\title{
Space and temporal evolution of density wave oscillations in multi-phase flow channels of boiling water reactors
}

\author{
J. L. Munoz-Cobo, A. Escrivá \& M. D. Domingo \\ Energy Engineering Institute, Universitat Politécnica de Valencia, Spain
}

\begin{abstract}
In this paper, a time dependent model for studying BWR in phase instabilities in the nonlinear regime is developed. This model is based on solving the mass energy and momentum conservation equations, for the single phase region and for both phases in the sub-cooled boiling and the bulk boiling regions. The model has been implemented in a code that also integrates: the recirculation loop dynamics equation; the heat transfer dynamic equations between the fuel and the channel; and the neutron kinetics equations. Special attention has been given in this paper to the sub-cooled boiling region and to the consequences of the degree of sub-cooling on the bubble dynamics. Also the direct heating of the water channel by neutrons and gamma rays has been considered. The result is a code called DYNAMICS that is able to perform in the time domain quantitative analysis of all the processes that affect the reactor stability.

Keywords: density wave oscillations, two phase flow channels, in phase oscillations in BWR, sub-cooled boiling.
\end{abstract}

\section{Introduction}

The problem of two phase flow instabilities it is found in a big variety of energy and chemical engineering systems such as the channels of Boiling Water Reactors (BWR), conventional steam boilers, and phase change heat exchangers used in the chemical industry. Among the different instability types the most important for the nuclear engineering field is the density wave oscillation mechanism (DWO), which is due to a multiple regenerative feedback between the mass flow rate, the steam generation rate, and the pressure drop [1, 2]. This phenomenon is especially interesting in BWR because there is a coupling via the 
void and Doppler reactivity feedbacks between the void fraction, and the fuel temperature with the core power. The goal of the present paper is to develop a time dependent model for studying BWR in phase instabilities in the nonlinear regime. This model is based on solving the mass energy and momentum conservation equations, for the single phase region and for both phases in the sub-cooled boiling and the bulk boiling regions. The model has been implemented in a code that also integrates: the recirculation loop equation, the heat transfer equations, and the neutron kinetics equations. Especial attention has been devoted to the sub-cooled boiling region and to the consequences of the degree of sub-cooling on the bubble dynamics, especially the effects due to the bubble collapse in the sub-cooled boiling region. Also the direct heating of the water channel by neutrons and gamma rays has been considered. The result is a code called DYNAMICS that is able to perform in the time domain quantitative analysis of all the processes that affect the reactor stability especially in the two phase region. The code results have been compared with stability measurement of BWR nuclear power plants at different stability conditions.

The paper is organized as follows, section 2 explains the thermal-hydraulic and neutronics models of the code, section 3 explains the discretization and integration of the equations, and section 4 contains the results for the steady state and an in-phase oscillatory reference case for a BWR reactor.

\section{The thermal-hydraulics and neutronic model in the DYNAMICS code}

\subsection{Channel thermal-hydraulics}

The actual model implemented in the DYNAMICS code is based on the integration of the mass, energy, and momentum equations in the single phase and two phase regions. Inside the two-phase region we distinguish between the subcooled boiling region and the bulk boiling region.

The mass conservation equations used in the sub-cooled boiling region (SBR) are the steam and the total mass ones. The steam mass conservation equation is:

$$
\frac{\partial}{\partial t}\left(\alpha \rho_{g}\right)=-\lambda_{s} \rho_{g} \alpha-\frac{1}{A} \frac{\partial}{\partial z}(A x G)+\frac{F_{s} Q_{c}^{\prime}}{A h_{f g}}
$$

where $F_{s}$ is the energy fraction transferred to the coolant that is invested in steam production, $\lambda_{s}$ is the rate of buble's destruction by collapsing in the sub-cooled coolant; $Q_{c}^{\prime}$ is the rate of energy transferred from the fuel to the coolant per unit length, $\mathrm{x}$ is the dynamic quality of the coolant, $\alpha$ the void fraction, $\mathrm{G}$ the mass flux, and A the channel area. In the bulk boiling region, we use for the steam the equation (1) with $\lambda_{s}=0$, and $F_{s}=1$. The conservation equation for the total mass is the same one in the sub-cooled and bulk boiling regions and is given by:

$$
\frac{\partial}{\partial t}\left(\alpha \rho_{g}+(1-\alpha) \rho_{l}\right)=-\frac{1}{A} \frac{\partial}{\partial z}(A x G)
$$


The conservation equation for the total energy of both phases, neglecting acoustic phenomena is in the sub-cooled and in the bulk boiling region given by:

$$
\frac{\partial}{\partial t}\left(\alpha \rho_{g} h_{g}+(1-\alpha) \rho_{l} h_{l}\right)=-\frac{1}{A} \frac{\partial}{\partial z}\left(A\left[(1-x) h_{l}+x h_{g}\right] G\right)+\frac{Q_{c}^{\prime}+Q_{D H}^{\prime}}{A}
$$

where $Q_{D H}^{\prime}$ is the rate of heat received per unit length by direct heating from neutrons and gamma rays. Because in equations (1) to (3) appears the quality and the void fraction, therefore we need a closure relation that relates the void fraction with the quality in both regions. The closure relation selected is [3]:

$$
\alpha=\frac{x}{x+s \frac{\rho_{g}}{\rho_{l}}(1-x)}
$$

where $\mathrm{s}$ is the slip ratio or ratio between the steam and liquid velocities. To compute $\mathrm{s}$ we use the modified Bankoff empirical correlation as determined by Jones and Digh [4], that is the same one used by the LAPUR code [5].

Concerning the momentum conservation equation for the two phase mixture in the channel is given by:

$$
\begin{aligned}
\frac{\partial}{\partial t} G= & -\frac{1}{A} \frac{\partial}{\partial z}\left[A\left\{\frac{(1-x)^{2}}{(1-\alpha) \rho_{l}}+\frac{x^{2}}{\alpha \rho_{g}}\right\} G^{2}\right]-\frac{\partial p}{\partial z}-\left((1-\alpha) \rho_{l}+\alpha \rho_{g}\right) g \\
& -f \Phi^{2} \Omega \frac{G^{2}}{2 \rho_{l} D_{h}}-K_{i n} \frac{G^{2}}{2 \rho_{l}}-\sum_{i} K_{i} \Phi_{i}^{2} \Omega_{i} \frac{G^{2}}{2 \rho_{l}} \delta\left(z-z_{i}\right)
\end{aligned}
$$

This equation takes into account the momentum changes due to: acceleration, pressure, gravity forces, friction forces, and pressure losses at the entrance and the spacers located at positions $z_{i}$. In equation (5), $\mathrm{G}$ is the mass flux, $\mathrm{f}$ is the channel wall friction factor; $\Phi^{2}$ is the two phase multiplier; $\Omega$ is the Jones multiplier; $\mathrm{D}_{\mathrm{h}}$ is the hydraulic diameter and $\mathrm{K}_{\mathrm{in}}$ denotes the form loss factor at the channel inlet. The last term in equation (5) takes into account the friction losses at the spacers, where $\mathrm{K}_{\mathrm{i}}$ denotes the form factor for the $\mathrm{i}$-th spacer.

\subsection{Heat transfer in the fuel}

We neglect in this case the axial conduction in the fuel because it is very small when compared with the radial conduction. Then starting from the heat transfer equation in the fuel rod and in the clad, it is obtained the following set of equations for the evolution of the average temperatures in the fuel and the clad respectively [6]:

$$
M_{f}^{\prime} c_{f} \frac{d \tilde{T}_{f}}{d t}=Q_{F}^{\prime}(t)-Q_{g}^{\prime}(t) \text {, and } M_{c l}^{\prime} c_{c l} \frac{d \widetilde{T}_{c l}}{d t}=Q_{g}^{\prime}(t)-Q_{c}^{\prime}(t)
$$

where $M_{f}^{\prime}$ is the fuel mass per unit length $(\mathrm{Kg} / \mathrm{m}), \mathrm{c}_{\mathrm{f}}$ is the fuel heat capacity coefficient $\left(\mathrm{J} / \mathrm{kg}{ }^{\circ} \mathrm{K}\right), M_{c l}^{\prime}$ is the clad mass per unit length, $\mathrm{c}_{\mathrm{cl}}$ is the heat capacity of the clad, $Q_{F}^{\prime}(t)$ is the heat generation rate in the fuel per unit length, 
$Q_{g}^{\prime}(t)$ is the heat transfer rate per unit length from the fuel to the clad. $\widetilde{T}_{f}$ and $\widetilde{T}_{c l}$ are the average temperatures in the fuel and the clad respectively.

In equation (6) the heat transfer rate per unit length from the fuel to the clad is given by the following expression [6]:

$$
Q_{g}^{\prime}(t)=\frac{n_{r o d s}}{R_{g}^{\prime}}\left(\widetilde{T}_{f}-\widetilde{T}_{c l}\right) \text { with } R_{g}^{\prime}=\frac{1}{8 \pi k_{f}}+\frac{1}{2 \pi r_{f} h_{g a p}}+\frac{1}{2 \pi k_{c l}}\left[\frac{r_{c l}^{2}}{r_{c l}^{2}-r_{f}^{2}} \ln \frac{r_{c l}}{r_{f}}-\frac{1}{2}\right]
$$

Being $R_{g}^{\prime}$ the thermal resistance per unit length from the fuel to the clad when using volumetric average temperatures and $\mathrm{h}_{\text {gap }}$ is the heat transfer coefficient of the gap. Finally the heat transfer rate per unit length from the clad to the coolant is given by :

$$
Q_{c}^{\prime}(t)=\frac{n_{r o d s}}{R_{c}^{\prime}}\left(\widetilde{T}_{c l}-\widetilde{T}_{c}\right) \text { with } R_{c}^{\prime}=\frac{1}{2 \pi r_{c l} h_{c}}+\frac{1}{2 \pi k_{c l}}\left[\frac{1}{2}-\frac{r_{f}^{2}}{r_{c l}^{2}-r_{f}^{2}} \ln \frac{r_{c l}}{r_{f}}\right]
$$

where $R_{c}^{\prime}$ is the thermal resistance per unit length from the clad to the coolant and $h_{c}$ the heat transfer coefficient from the clad surface to the coolant bulk.

\subsection{The point kinetics model}

The DYNAMICS-V1 code incorporates a pont kinetics model with one group of delayed neutrons and reactivity feedback by fuel temperature and void fraction. The kinetic equations used are the standard ones with one group of delayed neutron precursors. These equation for the neutron population $N(t)$ and the delayed neutron precursor population $C(t)$ are expressed in terms of the normalized variables: $n(t)=\left(N(t)-N_{0}\right) / N_{0}$ and $c(t)=\left(C(t)-C_{0}\right) / N_{0}$, where the subindex 0 means steady state values. On account of the previous definitions the standard point kinetic equation can be recasted in the form:

$$
\frac{d n}{d t}=\frac{\rho-\beta}{\Lambda} n+\frac{\rho}{\Lambda}+\lambda c, \text { and } \frac{d c}{d t}=\frac{\beta}{\Lambda} n-\lambda c
$$

where $\Lambda$ is the mean neutron generation time, $\lambda$ is the disintegration constant of delayed neutron precursors and $\beta$ is the total fractional yield of delayed neutron precursors.

The total feedback reactivity $\rho$ is composed of the void feedback reativity $\rho_{\alpha}$ plus the Doppler feedback reactivity $\rho_{D}=D_{f}\left(\widetilde{T}_{f}-\widetilde{T}_{f 0}\right)$, see reference [8].

To obtain the reactivity change due to the changes is the void fraction we take into account the void fraction values at each axial node, and on account of these void fraction values we compute the void fraction reactivity coefficients using the following expression: $\partial \rho_{\alpha} / \partial \alpha=c_{1}+c_{2} \alpha+c_{3} \alpha^{2}$, that depends on the void fraction at each particular node. Then, we multiply the reactivity coefficient at a given node by the void fraction variation in that node with respect to the steady state void fraction, finally we multiply the result by the reactivity weighting 
factor $\mathrm{W}_{\mathrm{r}, \mathrm{i}}$ for that particular node, that depends on the square of the power in the node, and we sum up for all the nodes [8].

The coupling of the fuel temperature equation with the point kinetic equations for the neutron populations is performed through the following relationship:

$$
Q_{F}^{\prime}(t)=\frac{P_{0}}{H} \frac{N(t)}{N_{0}}\left(1-f_{D H}\right)=\frac{P_{0}}{H}(1+n(t))\left(1-f_{D H}\right)
$$

where $\mathrm{P}_{0}$ is the steady state power, and $\mathrm{H}$ the active length of the reactor core, and $f_{D H}$ the fraction of energy deposited in the coolant by direct heating of neutrons and gamma rays. The amount of heat transfer rate per unit length to the coolant is given by the expression:

$$
Q_{c}^{\prime}=\frac{h A_{H T}}{H} \Delta T_{c}=U^{\prime} \Delta T_{c}
$$

where $\mathrm{h}$ is the average heat transfer coefficient from the clad to the coolant, $\mathrm{A}_{\mathrm{HT}}$ is the heat transfer area, and $\Delta T_{c}$ is the temperature difference between the clad and the coolant.

\subsection{Recirculation loop dynamics}

The recirculation loop is formed by the upper plenum, the steam separators, the downcomer, the jet pumps and the lower plenum. To simplify the calculations, the recirculation loop is treated as a a single path of uncompressible fluid and with variable area without boiling. With this assumption and integrating the mechanic balance equation from the loop inlet $\mathrm{s}_{\mathrm{iL}}$ to the loop outlet $\mathrm{s}_{\mathrm{oL}}$, it is obtained the following result, see reference [6] page 317 :

$$
\begin{aligned}
& \frac{1}{\bar{\rho}}\left(\frac{L}{A}\right) \frac{d W(t)}{d t}+\left(\frac{W(t)^{2}}{2}\right)\left(\frac{1}{\rho_{o L}^{2} A_{o L}^{2}}-\frac{1}{\rho_{i L}^{2} A_{i L}^{2}}\right)+\int_{s_{i L}}^{s_{o L}} \frac{1}{\rho(s)} \frac{\partial p}{\partial s} d s \\
&=-g\left(z_{o L}-z_{i L}\right)-\widetilde{E}_{v}+g H_{p}(W)
\end{aligned}
$$

where $\mathrm{W}(\mathrm{t})$ is the total mass flow rate $(\mathrm{kg} / \mathrm{s}) ; \mathrm{Hp}(\mathrm{W})$ is the manometric height of the pump; $\widetilde{E}_{v}$ is the coefficient of friction energy losses per unit mass; $\bar{\rho}$ is the average density of the coolant in the recirculation loop (RCL) and finally (L/A) is the ratio of length to area in the loop. These magnitudes are defined by:

$$
\Gamma=\left(\frac{L}{A}\right)=\bar{\rho} \int_{s_{i L}}^{s_{o L}} \frac{d s}{\rho(s) A(s)} \text {, and } \bar{\rho}=\frac{1}{V} \int_{s_{i L}}^{s_{o L}} \rho(s) A(s) d s
$$

Assuming that the energy loss rate per unit mass due to friction and area changes in the recirculation loop is given by $\widetilde{E}_{v}=K W^{2}$. Also we assume that for the case of transients where the coolant inventory remains constant in the recirculation loop, then the density gradients along this flow path are smalls in magnitude because it does not exist boiling along the flow path. Then it is a good approximation to assume that the third term of equation (12) is approximated by $\left(p_{o L}-p_{i L}\right) / \bar{\rho}$. 
Therefore from equation (12) and assuming that we are in natural circulation conditions, and it is possible to neglect the pump head effect, it is obtained after some simple manipulations the following equation for the mass flux variations with time at the channel entrance:

$$
\frac{d G(z=0, t)}{d t}=\frac{\delta p_{i L}(t)-\delta p_{0 L}(t)}{\Gamma A}+\frac{\Delta p_{f, n c, 0}}{\Gamma A}\left(1-\frac{G^{2}(z=0, t)}{G_{0}^{2}(z=0)}\right)
$$

where $\Delta p_{f, n c, 0}$ is the pressure drop by friction and form losses in the recirculation loop at steady state and natural circulation conditions, and $G(z=0, t)=W / A$ is the mass flux at the channel entrance. Finally $\delta p_{i L}(t), \delta p_{0 L}(t)$ are the pressure oscillations at the inlet and outlet of the recirculation loop with respect to the steady state pressures at natural circulation conditions i.e. $\delta p_{i L}(t)=p_{i L}(t)-p_{i L, 0}$, and $\delta p_{o L}(t)=p_{o L}(t)-p_{o L, 0}$.

\subsection{Closure relations and properties}

To closure the conservations equations displayed in the previous sections we need a set of empirical correlations, to obtain the numerical values of the model parameters as heat transfer coefficients, friction factors, two phase flow multipliers and so on.

The correction factor of Jones to the friction pressure drop in the two phase region is given by the expression: $\Omega=1.9-5 \times 10^{-4} \mathrm{G}$, being $\mathrm{G}$ the mass flux expressed in S.I. units of $\mathrm{kg} / \mathrm{m}^{2} \mathrm{~s}$.

For the local losses produced by the spacers we have used the multiplier expression obtained by Jones and Digh [9] that can be used for a broad range of pressure conditions. The expression for the Martinelli-Nelson multiplier used for the spacers depends on the pressure and the dynamic steam quality $\mathrm{x}$. A fit valid for $\mathrm{x}<0.7$ was synthetized by Jones and Digh [4] as:

$$
\Phi^{2}=\exp \left\{\sum_{i=1}^{4} a_{i}[\log (1+100 x)]^{i}\right\}
$$

where $\mathrm{a}_{\mathrm{i}}$ are pressure dependent fitted parameters given by:

$$
a_{i}=\sum_{j=1}^{8} b_{i j} p_{j}
$$

being $b_{\mathrm{ij}}$ fitting coefficients [9]; $p_{1}=1 ; p_{2}=1.42234 \times 10^{-2} p ; p_{j}=p_{2} p_{j-1}$ for $j=3, \ldots 6$, and finally $\mathrm{p}$ is the pressure in $\mathrm{kg} / \mathrm{cm}^{2}$. Because the spacers play an important role in reactor stability we have used expression (15) for the two-phase multiplier at the spacer location. To account for the variations in the MartinelliNelson multiplier produced by the void fraction variations, around the steady state conditions we have used the following Taylor expansion:

$$
\Phi^{2}=\Phi_{0}^{2}+\left(\frac{\partial \Phi^{2}}{\partial x}\right)_{0}\left(\frac{\partial x}{\partial \alpha}\right)_{0} \delta \alpha
$$


where $\delta \alpha=\alpha-\alpha_{0}$ is the void fraction variation with respect to the steady state value, and the partial derivatives have been obtained from equations (17), and the quality-void fraction relation.

The friction loss factor for the liquid has been obtained from the Moody correlation in the turbulent region $(\operatorname{Re}>2200), f_{l 0}=0.316 R e^{-0.25}$, being $\operatorname{Re}$ the Reynolds number at the channel conditions.

The heat transfer coefficients in the single-phase and two phase regions have been obtained using the Dittus-Boelter $\left(\mathrm{H}_{\mathrm{DB}}\right)$ heat transfer coefficient in the single-phase region, and the Jens-Lottes $\left(\mathrm{H}_{\mathrm{JL}}\right)$ heat transfer coefficient in the two phase region. These coefficients are given by the following expressions:

$$
\begin{gathered}
H_{D B}=\frac{k}{D_{h}} 0.023 \operatorname{Re}^{0.8} \operatorname{Pr}^{0.4} \\
H_{J L}=2.55528 \exp \left(6.4457 \times 10^{-7} p\right)\left(T_{w}-T_{\text {sat }}\right)^{3}=C_{2}\left(T_{w}-T_{\text {sat }}\right)^{3}
\end{gathered}
$$

where $\operatorname{Re}$ is the liquid Reynolds number, $\operatorname{Pr}$ is the Prandtl number, and $\mathrm{T}_{\mathrm{w}}$ is the clad wall temperature.

The procedure to determine the height of the inception point $\mathrm{z}_{1}$ for the subcooled boiling is obtained equating the single-phase forced convection heat flux, at the inception point to a Jens-Lottes type heat flux for two phase flow. The application of this criterion leads to the following expression for the liquid temperature $T_{l, z_{l}}$ at the inception [10], in terms of the heat flux $Q^{\prime \prime}$ at the wall:

$$
T_{l, z_{l}}=C_{2}^{-0.25} Q^{\prime \prime 0.25}+T_{s a t}-\frac{Q^{\prime \prime}}{H_{D B}}
$$

where $H_{D B}$ is the single-phase forced convection heat transfer coefficient, given by the Dittus-Boelter formula; $\mathrm{T}_{\text {sat }}$ is the saturation temperature, and the constant $\mathrm{C}_{2}$ is given by:

$$
C_{2}=2.55528 \exp \left(6.4457 \times 10^{-7} p\right)
$$

Below the inception point all the heat is invested in coolant heating, so $T_{1}(z)$ increases with $z$ along the vertical axis. Because the heating is not uniform the change in the liquid temperature must be determined on account of the power distribution along the channel. Then we must obtain the point $\mathrm{z}_{1}$ where the liquid temperature attains the inception temperature $T_{1, \mathrm{z} 1}$ given by equation (21) that depends on the heat flux and the fluid properties through the Re and Pr numbers. So an iterative procedure has been devised to obtain the height $z_{1}$ at which the inception point is located, in this iterative procedure we determine $T_{1}(z)$ at the lower nodes assuming all the heat is invested in liquid heating, then we get the inception temperature as a function of $z$, and this function decrease with $z$. Because $T_{1}(z)$ increase with $z$, we determine the point where $T_{1}(z)$, and $T_{1, z 1}(z)$ attain the same value. At this point the heat fluxes obtained from the DittusBoelter and the Jens-Lottes correlations are the same.

To obtain the heat fraction $F_{s}$ invested in steam production we take into account that the amount of heat transferred to the sub-cooled fluid can be decomposed according to Lahey and Moody [3] and Otaduy [5] in three 
components:

i) formation of steam bubbles near the heating surface which may detach into the main flow steam; ii) pumping of the liquid mass out of the control volume by the expanding action of the steam bubble formation; and (iii) single-phase convective heating through the parts of the heating surface no generating bubbles. The research performed on the importance of the three terms have concluded that the steam formation and the pumping process are predominant over the convective process to the liquid. Therefore on account of this previous discussion we can write $[3,10]$ :

$$
F_{s}=\frac{q_{\text {evap }}^{\prime \prime}}{q_{\text {evap }}^{\prime \prime}+q_{\text {pump }}^{\prime \prime}}=\frac{1}{1+\frac{q_{\text {pump }}^{\prime \prime}}{q_{\text {evap }}^{\prime \prime}}}
$$

where $q_{\text {evap }}^{\prime \prime}$ is the part of the energy flux related to the steam formation, while $q_{\text {pump }}^{\prime \prime}$ is the part of the energy flux associated to the pumping process. Now, according to Rouhani and Axelson [11], the quotient between both energy fluxes can be computed with the approximation that the liquid that leaves the control volume is at saturation, and therefore the quotient between both energy fluxes is:

$$
\varepsilon=\frac{q_{\text {pump }}^{\prime \prime}}{q_{\text {evap }}^{\prime \prime}}=\frac{j_{b} \rho_{f}\left(h_{f}-h_{l}\right)}{j_{b} \rho_{g} h_{f g}}=\frac{\rho_{f}}{\rho_{g}} H_{l} \Rightarrow F_{s}=\frac{1}{1+f_{p} \frac{\rho_{f}}{\rho_{g}} H_{l}}
$$

where $\mathrm{j}_{\mathrm{b}}$ is the steam volumetric flux and $\mathrm{H}_{1}$ is the degree of sub-cooling defined by: $H_{l}=\left(h_{f}-h_{l}\right) / h_{f g}$. Some codes as LAPUR include a correction factor $\mathrm{f}_{\mathrm{p}}=1.3$ to better correlate the predictions with the experimental data.

In the DYNAMICS program it was assumed that when the liquid enthalpy was close to the saturation value, then we were in the bulk boiling region and we assume that all the heat was invested in steam production.

To compute the decay constant for the void fraction in the sub-cooled region the model used by DYNAMICS is based on the Jones and Digh model [4], this model is also used by the LAPUR code [5]. The results of this model were compared with the expression used by the RELAP5 code [10], obtaining similar results. Jones model uses the following expression to calculate the time decay constant of the bubbles in the sub-cooled region:

$$
\lambda_{s}=c \lambda_{0} \phi H_{l}^{2}
$$

where $H_{l}$ is the degree of sub-cooling while $\phi$ and $\lambda_{0}$ are given by:

$$
\phi=\left(\frac{h_{f g}}{c_{p f}\left(T_{c 0}-T_{s a t}\right)}\right)^{2}, \text { and } \lambda_{0}=\frac{H_{w}^{2}}{k_{f} \rho_{l} c_{p f}}
$$

The constant $\mathrm{c}$ in equation (24) have taken equal to 0.125 , that it is a recommended value for the number of nodes used in the sub-cooled region for typical BWR channels. $\mathrm{T}_{\mathrm{c} 0}$ is the clad temperature at the inception of the sub- 
cooled boiling; $\mathrm{H}_{\mathrm{w}}$ is the single-phase heat transfer coefficient; $k_{f}$ is the liquid conductivity at saturation and finally $c_{p f}$ is the water specific heat at saturation.

\section{Discretization and integration of the equations}

\subsection{Discretization of the conservation equations}

The set of conservation equations (1), (2), (3) and (5) form a partial differential equation system. The integration of these equations with respect to the axial coordinate between the node limits produces a set of ordinary differential equations. Applying the operator $\frac{1}{\Delta} \int_{z_{i-1}}^{z_{i}} d z$ to these equations (1), (2), (3), (5) and defining the average nodal variables:

$$
\begin{gathered}
G_{i}(t)=\frac{1}{\Delta} \int_{z_{i-1}}^{z_{i}} d z G(z, t) ; \alpha_{i}(t)=\frac{1}{\Delta} \int_{z_{i-1}}^{z_{i}} d z \alpha(z, t) ; h_{l i}(t)=\frac{1}{\Delta} \int_{z_{i-1}}^{z_{i}} d z h_{l}(z, t) \\
p_{i}(t)=\frac{1}{\Delta} \int_{z_{i-1}}^{z_{i}} d z p(z, t) ; Q_{c, i}^{\prime}(t)=\frac{1}{\Delta_{i}} \int_{z_{i-1}}^{z_{i}} d z Q_{c}^{\prime}(z, t)
\end{gathered}
$$

It is obtained the following set of ordinary differential equations, that when the channel area is considered as constant reduce to:

$$
G_{i}\left(z_{i}, t\right)=G_{i}\left(z_{i-1}, t\right)+\Delta_{i}\left(\rho_{l i}-\rho_{g}\right) \frac{d \alpha_{i}}{d t}
$$

In the sub-cooled region the equation that gives the void fraction evolution is

$$
\frac{d \alpha_{i}}{d t}=\frac{F_{s i} Q_{c, i}^{\prime}}{\rho_{g} A h_{f g}}-\lambda_{s i} \alpha_{i}-\frac{1}{\Delta_{i} \rho_{g}}\left[x\left(z_{i}, t\right) G\left(z_{i}, t\right)-x\left(z_{i-1}, t\right) G\left(z_{i-1}, t\right)\right]=f_{s u b, i}
$$

The void fraction variation in the sub-cooled region can be also obtained from the following equation deduced from equations (27) and (28):

$$
\frac{d \alpha_{i}}{d t}=\frac{\frac{F_{s i} Q_{c, i}^{\prime}}{\rho_{g} A h_{f g}}-\lambda_{s i} \rho_{g} \alpha_{i}-\frac{1}{\Delta} G\left(z_{i-1}, t\right)\left[x\left(z_{i}, t\right)-x\left(z_{i-1}, t\right)\right]}{\left[\rho_{g}+\left(\rho_{l}-\rho_{g}\right) x\left(z_{i}\right)\right]}
$$

In the bulk boiling region from the discretized mass and energy conservation equations the following equation, for the void fraction evolution, is obtained:

$$
\frac{d \alpha_{i}}{d t}=\frac{\left(Q_{c, i}^{\prime}+Q_{D H, i}^{\prime} / A\right)+\frac{1}{\Delta_{i}}\left\{\left[x\left(z_{i}, t\right)-x\left(z_{i-1}, t\right)\right]\left(h_{f}-h_{g}\right) G\left(z_{i-1}, t\right)\right\}}{\rho_{g} h_{g}-\rho_{f} h_{f}+\left[h_{f}\left(1-x\left(z_{i}, t\right)\right)+h_{g} x\left(z_{i}, t\right)\right]\left(\rho_{f}-\rho_{g}\right)}
$$

In the sub-cooled-boiling region (SBR) and if the power oscillations are important, it is necessary to take into account the oscillations produced by the heat transfer on the liquid enthalpy. These evolution equations for the liquid enthalpy in the i-th node can be obtained from the energy conservation equation and are given by: 


$$
\begin{gathered}
\frac{d h_{l, i}}{d t}=-\frac{\left(\rho_{g} h_{g}-\rho_{l} h_{l, i}\right)}{\left(1-\alpha_{i}\right) \rho_{l, i}} f_{s u b, i}+\frac{Q_{c, i}^{\prime}+Q_{D H, i}^{\prime}}{\left(1-\alpha_{i}\right) \rho_{l, i}} \\
-\frac{\frac{1}{\Delta_{i}}\left[G\left(z_{i}, t\right)\left[\left(1-x\left(z_{i}\right)\right) h_{l}\left(z_{i}\right)+x\left(z_{i}\right) h_{g}\right]-G\left(z_{i-1}, t\right)\left[\left(1-x\left(z_{i-1}\right)\right) h_{l}\left(z_{i-1}\right)+x\left(z_{i}\right) h_{g}\right]\right.}{\left(1-\alpha_{i}\right) \rho_{l, i}}
\end{gathered}
$$

Finally the integration of the momentum equation along the the node, gives the following result for the pressure $p\left(z_{i-1}\right)$ at the lower node boundary in terms of the pressure $p\left(z_{i}\right)$ at the nodal upper boundary, when we have friction losses, and form losses at the entrace and the spacers:

$$
\begin{aligned}
p\left(z_{i-1}\right)= & p\left(z_{i}\right)+\Delta_{i} \frac{d G_{i}}{d t}+\left[M F\left(z_{i}\right)-M F\left(z_{i-1}\right)\right]+\Delta_{i}\left(\left(1-\alpha_{i}\right) \rho_{l}+\alpha_{i} \rho_{g}\right) g \\
& +\Delta_{i} F R_{i}+K_{0} \frac{G_{0}^{2}}{2 \rho_{l}} \delta_{i l}+\sum_{j} K_{j} \Phi_{j}^{2} \Omega_{j} \frac{G\left(z_{s p, j}\right)}{2 \rho_{l}}
\end{aligned}
$$

where $\operatorname{MF}\left(\mathrm{z}_{\mathrm{i}}\right)$ is the momentum flux at $\mathrm{z}=\mathrm{z}_{\mathrm{i}}$ :

$$
M F\left(z_{i}\right)=\left\{\frac{\left(1-x\left(z_{i}\right)\right)^{2}}{\left(1-\alpha\left(z_{i}\right)\right) \rho_{l}}+\frac{x\left(z_{i}\right)^{2}}{\alpha\left(z_{i}\right) \rho_{g}}\right\} G^{2}\left(z_{i}\right)
$$

$\mathrm{FR}_{\mathrm{i}}$ are the friction losses at node i per unit length, that are given by:

$$
F R_{i}=\frac{1}{\Delta_{i}} \int_{z_{i-1}}^{z_{i}} f \frac{\Phi^{2} \Omega}{2 \rho_{l} D_{h}} G^{2} d z \approx f_{i} \frac{\Phi_{i}^{2} \Omega}{2 \rho_{l} D_{h}}\left[\frac{G\left(z_{i}\right)+G\left(z_{i-1}\right)}{2}\right]^{2}
$$

In equation (32), it is necessary to compute $\Delta_{i} d G_{i} / d t$, to obtain the variation with time of the average mass flux in the node we assume that:

$$
\frac{d G_{i}(t)}{d t}=\frac{1}{2}\left[\frac{d G\left(z_{i-1}, t\right)}{d t}+\frac{d G\left(z_{i}, t\right)}{d t}\right]
$$

Now computing the time derivative of equation (27) with respect to time yields:

$$
\frac{d G\left(z_{i}, t\right)}{d t}=\frac{d G\left(z_{i-1}, t\right)}{d t}+\frac{\Delta_{i}}{2}\left(\rho_{l}-\rho_{g}\right) \frac{d^{2} \alpha_{i}}{d t^{2}}
$$

Therefore on account of (35) and (36) it is deduced that the average mass flux variation with time in node $\mathrm{i}$ can be expressed in terms of the flux variation and the channel entrance by means of the expression:

$$
\frac{d G_{i}}{d t}=\frac{d G_{0}}{d t}+\frac{1}{2}\left(\rho_{l}-\rho_{g}\right) \sum_{j=s b 1}^{i} \Delta_{i} \frac{d^{2} \alpha_{i}}{d t^{2}}
$$

where the sumation extends over all nodes located between the inception of subcooled boiling and the node $\mathrm{i}$.

\subsection{Integration of the equations and boundary conditions}

To integrate the set of equations (6), (9), (14), (27), (29), (30), (31) and (32), the first step is to solve the steady state equations i.e. with the time derivatives set to 
zero. To obtain the stationary solution we iterate until convergence is attained, in this way a set of variable values at steady state conditions are obtained and that are denoted by the sub-index 0 . Solving the steady state equations and using the constitutive relationships we get the steady state variables values, $\alpha_{i, 0}, i=1, . ., n d, G_{0}, \widetilde{T}_{f, 0}, \widetilde{T}_{c l, 0}, N_{0}, C_{0}, h_{l, i, 0}, i=1, \ldots n n d$. Where nnd denotes the number of axial nodes of the problem, typically 25 . Also we compute the steady state values of a set of magnitudes at the nodes boundaries: $p_{0}\left(z_{i}\right)$, $\alpha_{0}\left(z_{i}\right), x_{0}\left(z_{i}\right), s_{0}\left(z_{i}\right), u_{g, 0}\left(z_{i}\right), u_{l, 0}\left(z_{i}\right)$.

The boundary conditions that are applied to the model are:

1) It is assumed that the pressure at the upper plenum is known and is constant along time. This is a consequence of the assumption that there is not accumulation of steam and water in the upper plenum (UP) and this condition lead, as proved by Prassad et al. [2], to constant pressure in the upper plenum. March-Leuba [1] also considered this same boundary condition, but they assume that the UP pressure is mantained constant by the pressure regulator.

2) It is assumed that the coolant at the channel inlet of the core is sub-cooled and in liquid phase. Therefore at the channel inlet we have the following conditions: $\alpha(z=0, t)=0 ; T_{l}(z=0, t)=T_{i n}$.

3) The mass flux $G(z=0, t)$ at the channel inlet is determined by the dynamics of the recirculation loop.

Therefore solving the steady state equation we obtain the initial values of the problem at time 0 :

$$
\begin{aligned}
& \alpha_{i}(t=0)=\alpha_{i, 0}(z), G_{i}(t=0)=G_{0}, h_{l, i}(t=0)=h_{l, 0}, \widetilde{T}_{f}(t=0), \widetilde{T}_{c l a d}(t=0), \\
& p_{i}(t=0)=p_{i, 0}
\end{aligned}
$$

Then we substract from the ordinary diferential equations (ODE), their steady state equations and we obtain a set of ODEs for the variation of the magnitudes around their steady state values. These equations are denoted as the normalized equations. Obviously this set of ordinary differential equations is non linear and has been solved by a modified Runge-Kutta algorithm of 4-th order.

The numerical calculation of the set of equations during one time step proceed as follows, first it solves the set of differential equations system formed by: (i) equations (29) and (30) for the void fraction variations in the sub-cooled and the bulk boiling regions, (ii) equation (14) for the variations of the mass flux at the channel entrance, (iii) equations (6) for the average fuel temperature variations, and the average clad temperature variations, equations (9) for the neutron population normalized, and the delayed neutron precursors normalized and equations (31) for the sub-cooled enthalpy variations in the sub-cooled nodes. This previous set of equations when expresed in terms of its oscillations around their steady state values form a differential equation system of the form:

$$
\frac{\mathrm{d} \mathbf{y}}{\mathrm{dt}}=\mathrm{f}(\mathbf{y}, \mathbf{p}, \mathbf{G}, \mathrm{t})
$$

where the vector: $\boldsymbol{y}\left(\boldsymbol{\delta} \boldsymbol{\alpha}(t), \delta G_{0}(t), \delta T_{f}, \delta T_{c l}, n(t), c(t), \delta \boldsymbol{h}_{\boldsymbol{l}}(t)\right)$ denotes the magnitude variations around its steady state values. The vectors $\boldsymbol{G}(t)$ denotes the 
mass fluxes $\left(G_{1}(t), G_{2}(t), \ldots G_{n n d+1}(t)\right)$ at the node boundaries, obviously the mass flux at the first node boundary must be equal to the entrance to the channel mass flux denoted by $\mathrm{G}_{0}$. The vector $\boldsymbol{p}(t)$ denotes the pressures $\left(p_{1}(t), p_{2}(t), \ldots p_{n n d+l}(t)\right)$ at the boundaries of the channel nodes.

Simultaneously to the system of differential equations (38), we must solve the equations to obtain the must fluxes and the pressures at the nodes boundaries i.e. equations (27) and (32). In this case we note that in the previous equation (32) appears the term $\Delta_{i} d G_{i} / d t$, that is computed with the help of equation (37) and involves the calculation of $d^{2} \alpha_{i} / d t^{2}$.

The procedure during one time step $\mathrm{dt}$ is controlled by the subroutine DTGEN, that performs the following set of operations:

1) First the differential equation system (38) is integrated by means of a fourth step Runge-Kutta algorithm and using the pressure and mass flux values obtained in the previous time step, i.e. $G\left(z_{i}, t\right)$ y $p\left(z_{i}, t\right)$. This operation is performed at subroutine RK4N that calls to the function FCN that contains the system of ordinary differential equation (38), to be integrated. In this way we obtain the fluctuations of the state variable values at time $t+\Delta t$ i.e. $\boldsymbol{y}(\boldsymbol{t}+\Delta \boldsymbol{t})$.

2) The subroutine RK4N when it finish the standard fourth steps Runge-Kutta algorithm call to the subroutine FCN2, in this subroutine we use the values of the variables $\boldsymbol{y}(\boldsymbol{t}+\Delta \boldsymbol{t})$, to solve the equations (27) and (32). Proceeding in this way we update at $t+\Delta t$ the mass flux $G\left(z_{i}, t+\Delta t\right)$ and the pressure $p\left(z_{i}, t+\Delta t\right)$ at the node boundaries. At the end of these operations it is obtained the lower plenum pressure that will be used in the next time step for the integration of the recirculation loop equation. Then the new values are returned to the DTGEN subroutine that stores the computed values of all the state variables, and rename the variables to begin the calculation of a new time step.

\section{Dynamics results for a reference case}

To validate the dynamics model, we performed the reference case of the Vermont Yankee with the conditions of test $7 \mathrm{~N}$ (64\% Power, 32\% Mass Flow Rate), and $7^{\circ} \mathrm{C}$ of sub-cooling at the channel entrance, see references $[1,13]$. We assume to simplify the calculations that when the liquid enthalpy was close to the saturation value, then we assume bulk boiling conditions. At the boundary the fluid properties are the channel entrance boundary conditions i.e. $7^{\circ} \mathrm{C}$ of subcooling. We notice that the dynamic quality is different from zero at the boundary number 3 , or exit of the second node. This means that the inception for sub-cooling boiling takes places in node 2 , exactly at a heigh of $\mathrm{z}_{1}=15.28 \mathrm{~cm}$ inside the core active region. Because each node has a length of $14.26 \mathrm{~cm}$, then the inception of the sub-cooling boiling is located $1.06 \mathrm{~cm}$ above the beginning of the second node. 
The void fraction reactivity coefficients for the conditions of VY test $7 \mathrm{~N}$, were obtained by March-Leuba [12], and are given by the expression:

$$
\frac{d \rho_{\alpha}}{d \alpha}=-0.108-0.207 \alpha+0.140 \alpha^{2}-0.135 \alpha^{3}
$$

Using the boundary conditions for this test, it is observed the development of a limit cycle without using any multiplier for the reactivity. This means that the model predicts correctly the inception point of in-phase oscillations without any correction. Figure 1 displays the development of the limit cycle oscillations, the first 100 seconds are not displayed. Figure 2 displays the neutron population normalized i.e. $\mathrm{n}(\mathrm{t})$, versus the variations in the average (volume weighted) fuel temperature, $\delta \widetilde{T}_{f}(t)$, when the limit cycle is fully developped. We observe in figure 2, that $\mathrm{n}(\mathrm{t})$ and $\delta \tilde{T}_{f}(t)$, oscillate with some delay, i.e. when $\mathrm{n}(\mathrm{t})$ reach the maximum at $\mathrm{n}(\mathrm{t})=0.8$ then $\delta \widetilde{T}_{f}(t)=0$, and when $\delta \widetilde{T}_{f}(t)$ attain its maximum value then $n(t)=0$ according to figure 2 .

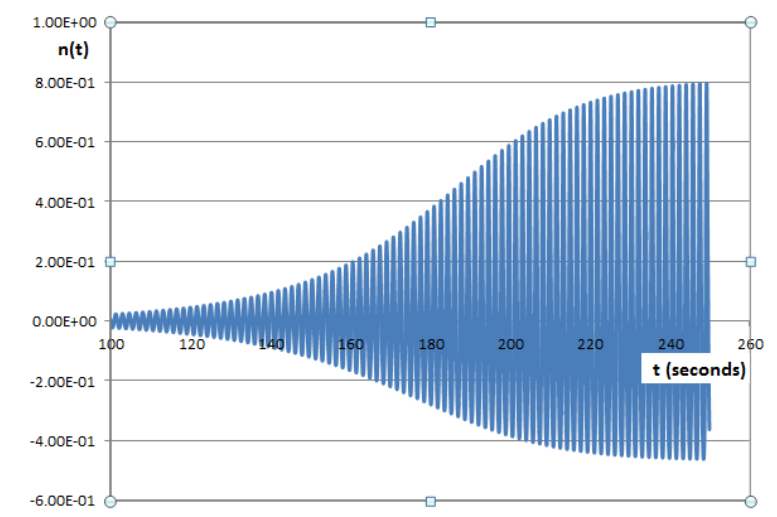

Figure 1: Development of a limit cycle for Vermont Yankee test $7 \mathrm{~N}$ model parameters and boundary conditions.

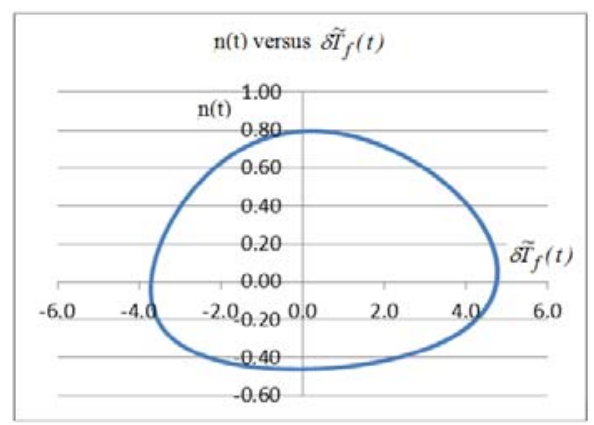

Figure 2: $\mathrm{n}(\mathrm{t})$ versus $\delta \widetilde{T}_{f}(t)$ when the limit cycle has been developed. 
It is observed that the void fraction in all the nodes oscillates in phase but with different amplitude, as displayed in figure 3. This last figure shows the void fraction variations $\delta \alpha_{i}(t)$, at the first two boiling nodes, located after the subcooled boiling inception point. The oscillation with smaller amplitude is for the node with the water more sub-cooled.

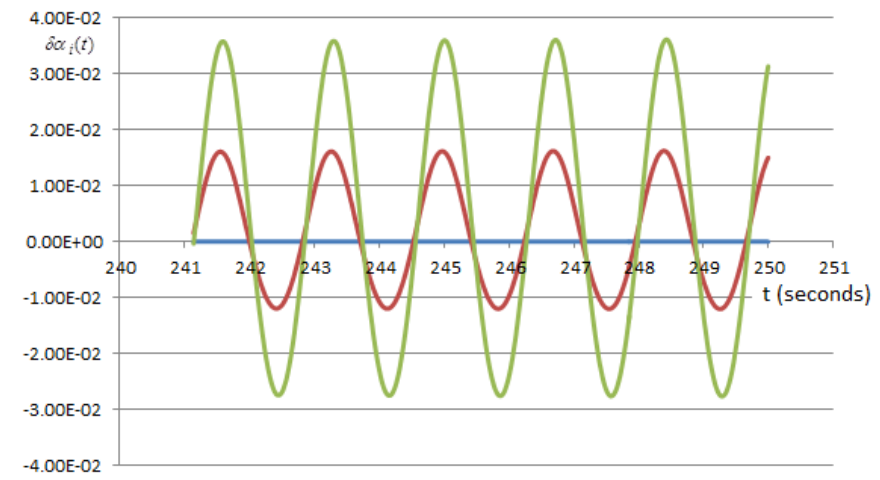

Figure 3: $\delta \alpha_{i}(t)$ versus $t$ for the first two nodes with boiling.

\section{Conclusions}

The development of a two-phase flow thermal-hydraulic model to study the inphase instabilities taking into account the spatial effects along the channel and the sub-cooled boiling has two main difficulties first we need to obtain the inception point for the sub-cooled boiling, and also to determine the bulk boiling boundary, and second the dynamic equations in the sub-cooled boiling region and the bulk boiling region are different. Also we must take into account that in the sub-cooled boiling region the liquid is not saturated and therefore during a limit cycle the liquid enthalpy can oscillate. However if the amplitude of the oscillations is small, then one can neglect the oscillation in the enthalpy in the sub-cooled boiling nodes, this approximation cannot be performed for large power oscillations.

We have checked that increasing the sub-cooling of the liquid at the channel entrance with this model the reactor becomes more stable for in-phase oscillations, and we need more reactivity gain to attain limit cycle oscillations. Also we have obtained that the direct coolant gamma heating has some influence in the decay ratio that cannot be neglected. These in-phase oscillations have a frequency between 0.38 and $0.5 \mathrm{hz}$ depending on the boundary conditions.

The maximum amplitude observed for the normalized power oscillations for the reference case, is of 0.8 , that for the reference case of $64 \%$ of rated power is equivalent to a power oscillation amplitude of $116 \%$, because the scram level is not crossed, the limit cycle is developed at this operating conditions of $\mathrm{P}_{0}=64 \%$ of the rated power and $\mathrm{G}_{0}=32 \%$ of the rated flow. 


\section{Acknowledgement}

The authors are indebted to the help received from the Plan Nacional I+D ENE2013-48565-C2-1.

\section{References}

[1] March-Leuba, J., "Dynamic Behaviour of Boiling Water Reactors", Thesis University of Tennessee, 1984.

[2] Prassad, R.O.S., Doshi, J.B., \& Iyer, K., "A numerical Investigation of Nuclear Coupled Density Wave Oscillations”, Nucl. Eng. Design 154, pp. 381-396 (1995).

[3] Lahey, R.T., \& Moody, F.J., "The Thermal-hydraulics of Boiling Water Reactors", Published by the ANS (1993).

[4] Jones, A.B., \& Digh, D.G., "Hydrodynamic Stability of a Boiling Channel" Report KAPL-2290 (1963).

[5] Otaduy, P.J., "Modeling of the dynamic behavior of large BWR cores". PhD Thesis University of Florida (1979).

[6] Lewis, E., "Nuclear Power Reactor Safety". John Wiley and Sons (1977).

[7] Lewins, J.D., "Nuclear Kinetics and Control”, Pergamon Press (1989).

[8] Muñoz-Cobo J.L., Escriva, A., \& Garcia, C. "Consistent generation of point feedback reactivity parameters for stability and thermal-hydraulic codes”, Annals of Nuclear Energy vol. 33, pp. 1147-1156 (2006).

[9] Jones, A.B., \& Digh, D.G., "Hydrodynamic Stability of a Boiling Channel" Report KAPL-2208 (1962).

[10] Muñoz-Cobo, J.L., Chiva, S., \& Sekhri, A., "A reduced order model of BWR dynamics with sub-cooled boiling and modal kinetics: application to out of phase oscillations", Annals of Nuclear Energy, Vol 31, pp. 11351162 (2004).

[11] Rouhani, S.Z., \& Axelson, E., "Calculation of void fraction volume in sub-cooled and quality boiling region" Int. Journal of Heat and Mass Transfer 13, pp. 383-393 (1970).

[12] March-Leuba, J., Cacucci, D.G., \& Pérez, R.B., "Non Linear Dynamics and Stability of BWR Part 2, Quantitative Analysis", Nuclear Science and Engineering 93, pp. 124-136 (1986). 